\title{
Signal convergence in fruits: a result of selection by frugivores?
}

\author{
S. B. LOMÁSCOLO*1 \& H. M. SCHAEFER† \\ *Department of Zoology, University of Florida, Gainesville, FL, USA \\ $\dagger$ †epartment of Animal Ecology and Evolutionary Biology, Faculty of Biology, University of Freiburg, Freiburg, Germany
}

Keywords:

colour vision model;

Dispersal syndromes;

frugivory;

plant-animal communication;

signal evolution.

\begin{abstract}
The Dispersal Syndrome hypothesis remains contentious, stating that apparently nonrandom associations of fruit characteristics result from selection by seed dispersers. We examine a key assumption under this hypothesis, i.e. that fruit traits can be used as reliable signals by frugivores. We first test this assumption by looking at whether fruit colour allows birds and primates to distinguish between fruits commonly dispersed by birds or primates. Second, we test whether the colours of fruits dispersed by primates are more contrasting to primates than the colours of bird-dispersed fruits, expected if fruit colour is an adaptation to facilitate the detection by seed dispersers. Third, we test whether fruit colour has converged in unrelated plant species dispersed by similar frugivores. We use vision models based on peak sensitivities of birds' and primates' cone cells. We base our analyses on the visual systems of two types of birds (violet and ultraviolet based) and three types of primates (trichromatic primates from the Old and the New Worlds, and a dichromatic New World monkey). Using a Discriminant Function Analysis, we find that all frugivore groups can reliably discriminate between bird- and primatedispersed fruits. Fruit colour can be a reliable signal to different seed dispersers. However, the colours of primate-dispersed fruits are less contrasting to primates than those of bird-dispersed fruits. Fruit colour convergence in unrelated plants is independent of phylogeny and can be better explained by disperser type, which supports the hypothesis that frugivores are important in fruit evolution. We discuss adaptive and nonadaptive hypotheses that can potentially explain the pattern we found.
\end{abstract}

\section{Introduction}

To overcome the constraints imposed by immobility, many plants depend on animals for dispersal of pollen and seeds. Animal pollinators and dispersers cue on plant signals (colour and odour) to find flowers and ripe fruits. Yet the great diversity of plant odours and colours is poorly understood - it has been variously attributed to chance, to environmental control, and to the sensory ecology of mutualistic animals (Ben-Tal \& King, 1997;

Correspondence: Silvia B. Lomáscolo, Department of Zoology, University of Florida, Gainesville, FL 32611-8525, USA.

Tel.: 54261524 4166; fax: 54261524 4001;

e-mail: slomascolo@gmail.com

${ }^{1}$ Present address: Laboratorio de Interacciones Ecológicas, IADIZA,

CRICYT, 5500 Mendoza, Argentina.
Gumbert et al., 1999; Johnson \& Steiner, 2000). The latter, in particular, constitutes a long-debated central question in the field of plant-animal interactions: Do animal mutualists consistently, and differentially, select plant traits, resulting in trait convergence into pollination and seed dispersal syndromes (Ridley, 1930; van der Pijl, 1961, 1969; Janson, 1983; Johnson \& Steiner, 2000)? Syndromes are sets of flower or fruit traits hypothesized to evolve under the selective pressure of pollinators (Pollination Syndrome hypothesis) and seed dispersers (Dispersal Syndrome hypothesis), respectively. Syndromes result from the convergence of floral or fruit traits in unrelated plants sharing the same suites of visitors, which may be adaptive, because visitation rate is commonly dependent on resource density (Feinsinger et al., 1991; Ben-Tal \& King, 1997; Forsyth, 2003). This 
'Signal Convergence hypothesis' is the main focus of this study. We test for the convergence of one trait, fruit colour, that is very important to define dispersal syndromes, because frugivores commonly use it as a signal to find and asses fruits (Schmidt et al., 2004; Voigt et al., 2004; Burns, 2005; Schaefer et al., 2006, 2008a).

Nevertheless, the general convergence of fruit or flower traits to syndromes that are reliably associated with (i.e. presumably signal to) specific animal vectors has been questioned. Detractors of the syndrome hypotheses argue that plants tend to benefit from different types of animal mutualists and, conversely, plant-visiting animals tend not to specialize on only one type of plant (Herrera, 1985; Waser et al., 1996; Johnson \& Steiner, 2000). This argument is particularly relevant to the evolution of seed dispersal syndromes, because plants show a lower degree of specialization towards seed dispersers than they do towards pollinators (Blüthgen et al., 2007). From an adaptive viewpoint, the low degree of specialization in seed dispersal will result in disparate selective pressures on fruit appearance and will, thus, reduce the likelihood of signal convergence. Some authors claim that apparently nonrandom combinations of fruit traits are evidence of syndromes (Janson, 1983; Knight \& Siegfried, 1983; Gautier-Hion et al., 1985; Willson et al., 1989; Kalko et al., 1996; Korine et al., 2000). However, when those combinations of traits have been scrutinized in phylogenetically controlled studies, the syndromes are generally not supported (Herrera, 1987; Fischer \& Chapman, 1993). This difference in approach and opinion has generated much controversy.

We argue that one reason studies addressing the role of frugivores in shaping fruit evolution have reached disparate conclusions is that fruit colour, one of the most important fruit traits reflecting differential food selection by distinct frugivore assemblages (Voigt et al., 2004), has been measured inappropriately. Colour as a visual cue might be particularly important in defining syndromes, because it represents the first step in the interactions between plants and animals. Natural selection should favour signals (fruit colours) that elicit a response in the receiver (the frugivore) that increases the fitness of the sender (the plant) (Endler, 1992). From this, we can derive that the efficiency of a signal depends not only on the signal's form (i.e. the spectral reflectance of the fruit), but also on the receiver's perception (i.e. the frugivore's neural circuitry to interpret the colour signal) (Endler, 1992). Nonetheless, evolutionary studies on fruits and frugivores have typically reported colour qualitatively and according to human visual perception (Janson, 1983; Gautier-Hion et al., 1985; Fischer \& Chapman, 1993; Lord et al., 2002), or they have ignored colour (Jordano, 1995), probably because of the difficulty of quantifying it.

Another important property of signals is their conspicuousness which is quantified as the signal-to-noise ratio. In the case of visual signals, conspicuousness is directly correlated with the contrast between the colour of the object and that of the surrounding environment. In general, receivers are assumed to select for maximum conspicuousness of the signal. In the case of fruits, the contrast between the colour of the fruit and the foliage background should be greatest to the disperser to which the signal is targeted. Although fruit conspicuousness has been shown to be important for fruit detection (Sumner \& Mollon, 2000a; Regan et al., 2001; Burns \& Dalen, 2002; Schmidt et al., 2004), the contrast between the colour of the fruit and that of its background has never been incorporated in a study of dispersal syndromes.

Our study focuses on the role of fruit colour as a signal to two of the most common diurnal frugivorous taxa, primates and birds, which differ in their visual abilities (Hastad and Ödeen 2008). One assumption of our study is that primates and birds can have differential effects on plant fitness according to their differences in seed treatment and recruitment requirements of different plants. Although support is scant, some published studies suggest that this is a fair assumption (Clark et al., 2001; Poulsen et al., 2002). Under the Signal Convergence hypothesis, we test whether fruit colour can be used as a signal to seed dispersers. We predict the followings:

1. Primates should be able to distinguish visually between fruits that are primarily dispersed by them and those that are primarily dispersed by birds. Birds should do the same.

2. Given that the visual systems of birds and primates differ, fruit colours should be more conspicuous to the disperser (primates or birds) to which they are signalling than to other dispersers.

3. The colour of fruits dispersed mainly by primates should converge to colours that are different from those that are dispersed mainly by birds.

To test prediction no. 1 of the Signal Convergence hypothesis, we use vision models to analyse fruit signals according to the colour vision capabilities of primates and birds (Osorio \& Vorobyev, 1996; Vorobyev \& Osorio, 1998; Regan et al., 2001; Endler \& Mielke, 2005). To test prediction no. 2, we define conspicuousness as the contrast in colour between the fruit and the foliage background, and we use vision models to calculate conspicuousness of fruits to each frugivore type. To test prediction no. 3, we perform a phylogenetic comparative analysis to see whether color discrimination by birds and primates can be explained by phylogenetic relations of plants. For no. 1 and 2 predictions, we used the largest data set of spectral fruit colours analysed to this date. For 402 plant species with known fruit consumers, we demonstrate that primates are physiologically capable of discriminating between fruits dispersed by them and those dispersed by birds based solely on colour. Likewise, we show that birds can do the same and that this pattern transcends visual capability of different frugivores, geographic areas and persists even when phylogenetic effects 
are controlled. Prediction no. 3 was tested with 187 species for which phylogenetic information was available. We also discuss why fruit colours were not always more conspicuous to the targeted disperser.

\section{Materials and methods}

\section{Fruit colour and disperser data}

Data on reflectance spectra of fruits and data on fruit consumer for each plant species were obtained mainly from the literature and partly from our fieldwork. A total of 402 species were included, of which 176 were primatedispersed species from French Guiana (110) (Regan et al., 2001), Uganda (46) (Sumner \& Mollon, 2000b) and Venezuela (20) (Schmidt et al., 2004), and 226 were birddispersed species from Florida (28) (Schaefer et al., 2007), Europe (99) (Schaefer et al., 2007), Venezuela (31) (Schmidt et al., 2004) and other regions (68) (Schaefer et al., 2007, and fieldwork). These authors investigated how fruit colours are perceived by either birds or primates, which resulted in exclusive categories of dispersers. All data on dispersers were based on observations at fruiting plants by these authors. We made sure that disperser was never assigned based on fruit morphology, as that would have incorporated circularity in our analyses. In all cases, colour was quantified using a portable spectrometer and an external light source. Fruit reflectance was obtained as the proportion of reflectance against a white standard. The spectrometer, light and white standard varied among studies but were consistent within each study. Although the use of different equipment adds an uncontrolled source of variation in the data, we considered that, if the predicted patterns were found in reflectance of primate- vs. bird-dispersed fruits, this would be despite the lack of consistence in colourmeasuring devices, and not because of it. Notwithstanding, we still took measures to make sure that the inclusion of studies with different methodologies did not affect our results (see 'Data Analysis: Prediction no. 1 - Colour discrimination by birds and primates' for more details).

Spectra were averaged in 5-nm intervals from 400 to $700 \mathrm{~nm}$. Unfortunately, data on fruit reflectance under $400 \mathrm{~nm}$ (the UV range) were not available for most primate-dispersed fruits, so we left this part of the visual range of birds out of our main analyses. Our results are, therefore, conservative, because differences between fruit colour perception by primates and birds are likely more pronounced if UV vision is accounted for. Nonetheless, to test whether our conclusions held if we had included reflectance in the UV range, we repeated the analysis with a smaller data set consisting of nine primate- and 68 bird-dispersed fruits for which reflectance in the UV range was available. All of these fruits belonged to different genera to minimize potential phylogenetic biases.

\section{Vision model}

As colour is interpreted by the brain based on the differential input of light to cone cell types with different sensitivity to specific wavelengths (Regan et al., 2001; Bowmaker \& Hunt, 2006), we calculated the light input to the different cone types for each frugivore group. Our vision model combines the reflectance spectrum of each fruit with the colour vision ability for each frugivore and yields three variables per species of fruit, corresponding to the quantum catch of the short-, medium- and longwavelength cones of the frugivore (Fig. 1). For primates, we used three different models to account for the variation in spectral sensitivities among primates. We modelled cone peak sensitivities corresponding to humans (440, 540 and $570 \mathrm{~nm})$, which represent Old World primates and are very similar to those of other trichromatic primates (e.g. Alouatta spp.), to trichromatic Tufted capuchins (Cebus apella) (430, 536 and $562 \mathrm{~nm}$ ) and to dichromatic Tamarins (Sanguinus sp.) (430 and $443 \mathrm{~nm}$ ) (Regan et al., 2001; Bowmaker \& Hunt, 2006). Birds are less variable in their spectral sensitivities than primates. We used two models that characterize the two major classes, one with the short-wavelength sensitivity biased towards the violet (hereafter VS-birds) and one biased towards the ultraviolet (hereafter UV-birds) (Ödeen \& Hastad, 2003). The cone peak sensitivities of the VS-birds $(416,478,542$ and $607 \mathrm{~nm}$ ) are given in Endler \& Mielke (2005), and those of the UV-birds are represented by those of the Blue Tit (Cyanistes caeruleus, 372, 456, 544 and $609 \mathrm{~nm}$ ). As mentioned earlier, the published data on reflectance spectra were limited to reflectance between 400 and $700 \mathrm{~nm}$, and hence, sensory input to the cones with sensitivity to the shortest wavelength was out of the range for UV-birds and too close to the limit for VS-birds. Hence, for both types of birds, we only included in the model the sensitivity to the other three cones. In each model, we calculated the relative cone excitation values, called quantum catches (Fig. 1), which represent the basic sensory input for colour vision (Kelber et al., 2003).

All cone excitation values were transformed using the natural logarithm to achieve normality and were analysed using Discriminant Function Analysis (DFA) to test whether bird-dispersed fruits and primate-dispersed fruits were significantly discriminated in all visual systems using the quantum catch of bird and primate cones in five separate analyses. Discriminant Function Analysis finds the combination of the variables that best discriminates between the groups (bird- vs. primatedispersed fruits) and identifies each variable's contribution to the discrimination. These combinations of variables are called Linear Discriminant Functions (LDF). If birds can visually discriminate between fruits commonly dispersed by them and those commonly dispersed by primates, a significant discrimination should be 

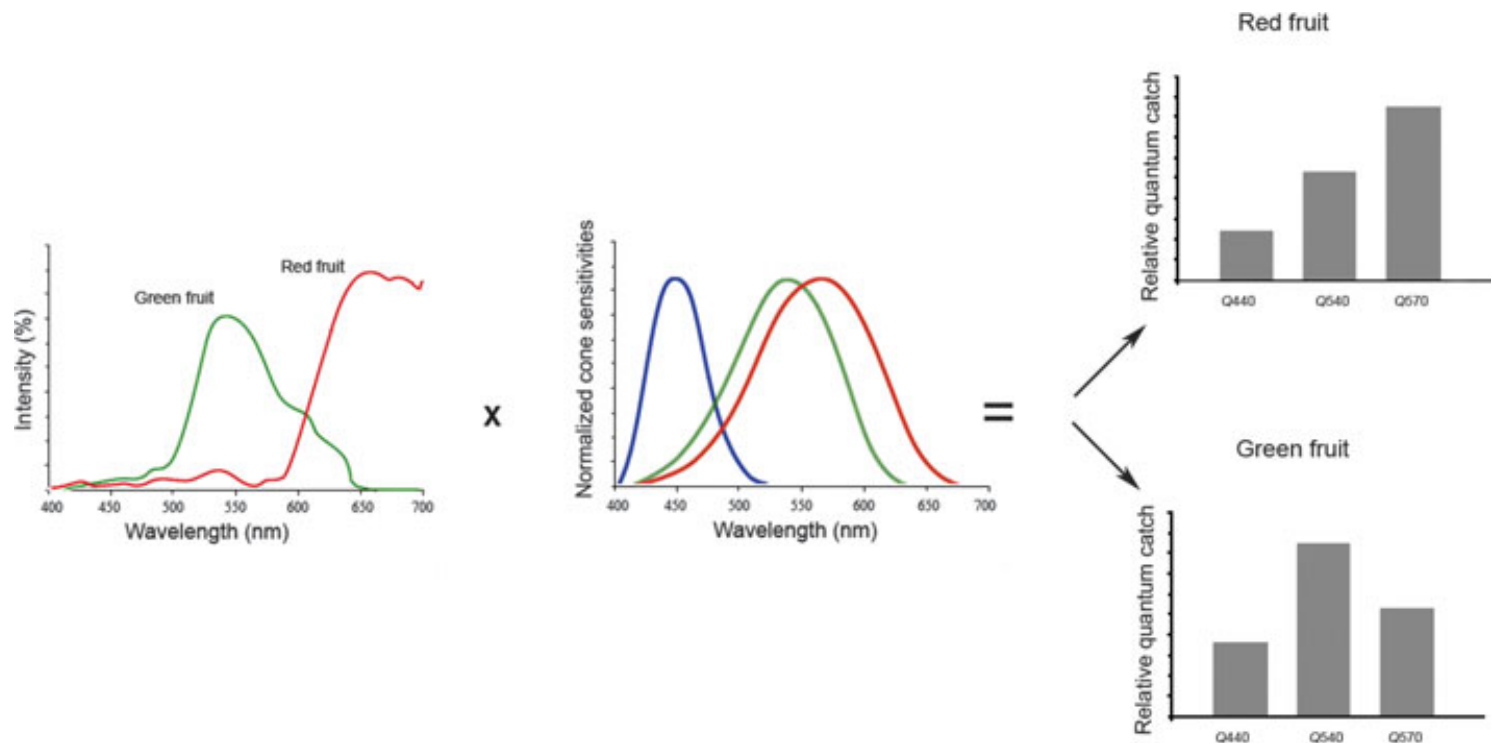

Fig. 1 Reflectance spectra of two different fruits (left) combined with the peak cone sensitivities of a frugivore (middle) yields the quantum catch values (Q440, Q540, Q570) for the three cone types for each fruit (right). Quantum catch values approximate the frugivore's perception of colour.

obtained using the basic sensory input of bird vision. Likewise, the same should be obtained for primates.

\section{Data analysis}

All data analysis was carried out using R 2.6.0 and 2.9.0 (R Development Core Team, 2008), with the exception of Evolutionary Principal Components Analysis (see later) which is implemented in Mesquite 2.01 (Dyreson $\delta$ Maddison, 2006; Maddison \& Maddison, 2007). Phylogenetic reconstruction of our species was performed using Phylomatic (Campbell \& Donoghue, 2004). All software is freely available online.

\section{Prediction no. 1 - colour discrimination by birds and primates}

To evaluate the accuracy of the DFA and its predictive power, we split our data set of 402 species into two parts and derived the LDFs with one part (206 species from French Guiana, Florida and other varied geographic regions), called the 'training' DFA and tested the predictive value of LDFs with a validation DFA performed on a completely independent data set formed by the other part of our data, (196 species from Uganda, Europe and Venezuela). This is a powerful test (see Sharma et al., 2005) of the validity of the discrimination between primate and bird fruits by primate and bird vision, because the pattern of discrimination will remain significant only if it is consistent across very different biogeographical regions, such as the Palaeotropics, Palaeo-temperate areas and the Neotropics. We also tested the predictive value of the LDFs by classifying the cases as 'bird' or 'primate' fruits based on their score on the LDF derived with the 'training' DFA. The higher the percentage of species that were well classified (known bird-dispersed species correctly classified as a birddispersed species), the higher the predictive value is of the LDFs. This was carried out separately for the 'training' and the validation data sets.

Variation in geography as well as in the methods used to obtain fruit colour spectra may introduce biases in our data set. To make sure that our results were not affected by the distribution of species into the 'training' and the validation data sets, we tested several other combinations of species in both data sets, including different random samples where half of the data from each region were included in the 'training' set, and the other half in the validation set. As the results do not change qualitatively, we only report the results of the analysis described previously.

\section{Prediction no. 2 - conspicuousness of the fruits to primates}

To test whether primate-dispersed fruits contrasted more against the foliage than bird-dispersed fruits from a primate's visual perspective, we first calculated the chromatic contrasts of 86 primate fruits and 188 bird fruits against their species-specific foliage background as the Euclidean distance between the colour of a fruit and the colour of the structure against which it is viewed by the frugivore. We restricted this analysis to the subset of 256 species for which species-specific background information was available. The resulting contrasts for primate and bird fruits were entered into the primatevision model, and the results were compared using a Mann-Whitney $U$-test. Unfortunately, we could not test 
conspicuousness of fruits to birds, because UV reflectance is required to obtain an accurate measure of conspicuousness to birds. To ensure that the differences between fruit types are actually attributed to the differences in reflectance by the fruit itself and not by the background, we also calculated fruit contrasts against the mean background for the 256 species included in the analysis and compared the results using another Mann-Whitney $U$-test.

\section{Prediction no. 3 - phylogenetic control}

Closely related species are not independent data points, because they share a common ancestor. Our specific question here is whether the discrimination by birds and by primates between bird- and primate-dispersed species can be explained by the phylogeny of our plant taxa. Hence, we did a comparative phylogenetic analysis based on a phylogeny of the plant species included in this study, which was constructed using a free online program called Phylomatic (Campbell \& Donoghue, 2004). This software builds the phylogeny based on master trees in their data base, called 'megatrees'. Only 187 species were included in the phylogeny because, first, not all species are included in the database used by Phylomatic and, second, because Phylomatic only resolves trees to the genus level (Fig. S1). That means that all species belonging to the same genus are coded as a polytomy, which is uninformative for our comparative phylogenetic analysis. Hence, we included only one species of each genus in the tree, randomly chosen using a random number generating algorithm in R (R Development Core Team, 2008). We controlled for phylogenetic effects by doing a Canonical Phylogenetic Ordination (Giannini, 2003; Herder et al., 2006; de Pinho Werneck et al., 2009). This method seemed particularly appropriate for two reasons: first, it subdivides the phylogenetic tree into clades, which takes into account that not all clades may be related to the pattern in the variable in question and allows for a more detailed analysis of which specific clades have a stronger phylogenetic signal; and second, it is strictly based on tree topology and not on ancestral character state reconstruction, so fewer assumptions are made when inferring the phylogenetic structure of the comparative data (Giannini, 2003).

This method is based on a general regression model of the form

$$
Y=\beta X+\xi
$$

where $Y$ represents the comparative data, in this case, the score of each plant species on the LDF, $\beta$ is a regression coefficient of the $Y-X$ relationship, $X$ represents membership to the different monophyletic clades in the phylogenetic tree, i.e. the phylogenetic tree structure, and $\xi$ is an error term. The matrix of group membership was generated using the program TNT (Goloboff et al., 2008) and is of the simple form

\begin{tabular}{llll}
\hline & Clade 1 & Clade 2 & Clade 3 \\
\hline Species 1 & 1 & 1 & 0 \\
Species 2 & 1 & 1 & 0 \\
Species 3 & 0 & 1 & 0 \\
Species 4 & 0 & 1 & 0 \\
Species 5 & 0 & 0 & 1 \\
Species 6 & 0 & 0 & 1 \\
\hline
\end{tabular}

where clade membership is represented by l's.

First, a regression is run between each variable $X$ representing each monophyletic clade in the tree and the dependent variable, i.e. the score on the LDF generated by doing a Discriminant Analysis with the 187 species included in the tree. To prevent inflating the significant results because of the multiple comparisons carried out, we used a sequential Bonferroni correction (Rice, 1989) to determine all clades that significantly explain the variation in $Y$. Second, all the significant clades were used to find the best model that explains the variation in $Y$. This was carried out by using a General Linear Model approach using the glm procedure in R 2.9.0 (R Development Core Team, 2008). In summary, if the observed pattern of discrimination is independent of phylogeny, then clade membership should not affect the score of each species on the LDF. Hence, we should find that none of the $X$ variables significantly explain the dependent $Y$ variable. If, instead, phylogeny is important in defining the pattern of discrimination that we find, we will find that many clades explain the dependent variable $Y$, and we can identify which clades have the strongest phylogenetic signal. Another method based on character state reconstruction called Evolutionary Principal Components analysis (EPCA) (Dyreson \& Maddison, 2006; SchlickSteiner et al., 2006) gave qualitatively similar results, which are presented in the Supporting Information.

\section{Results}

\section{Prediction no. 1 - colour discrimination by birds and primates}

The 'training' DFA resulted in significant discrimination between bird- and primate-dispersed fruits when using both types of bird cone sensitivity peaks (VS-birds: Wilk's $\lambda=0.645$, numerator d.f. $=3$, denominator d.f. $=$ 202, $P<0.00001$; UV-Birds: Wilk's $\lambda=0.661$, numerator d.f. $=3$, denominator d.f. $=202, P<0.00001)$ and all three primate cone sensitivity peaks (humans: Wilk's $\lambda=0.655$, numerator d.f. $=3$, denominator d.f. $=202, \quad P<0.00001$; dichromatic tamarins: Wilk's $\lambda=0.703$, numerator d.f. $=2$, denominator d.f. $=203$, $P<0.00001$; Tufted capuchins: Wilk's $\lambda=0.684$, numerator d.f. $=3$, denominator d.f. $=202, \quad P<0.00001$ ) (Fig. 2). For all different distributions of species in the 'training' and validation sets tested, discrimination was significant (all $P$-values < 0.00001). Classification 

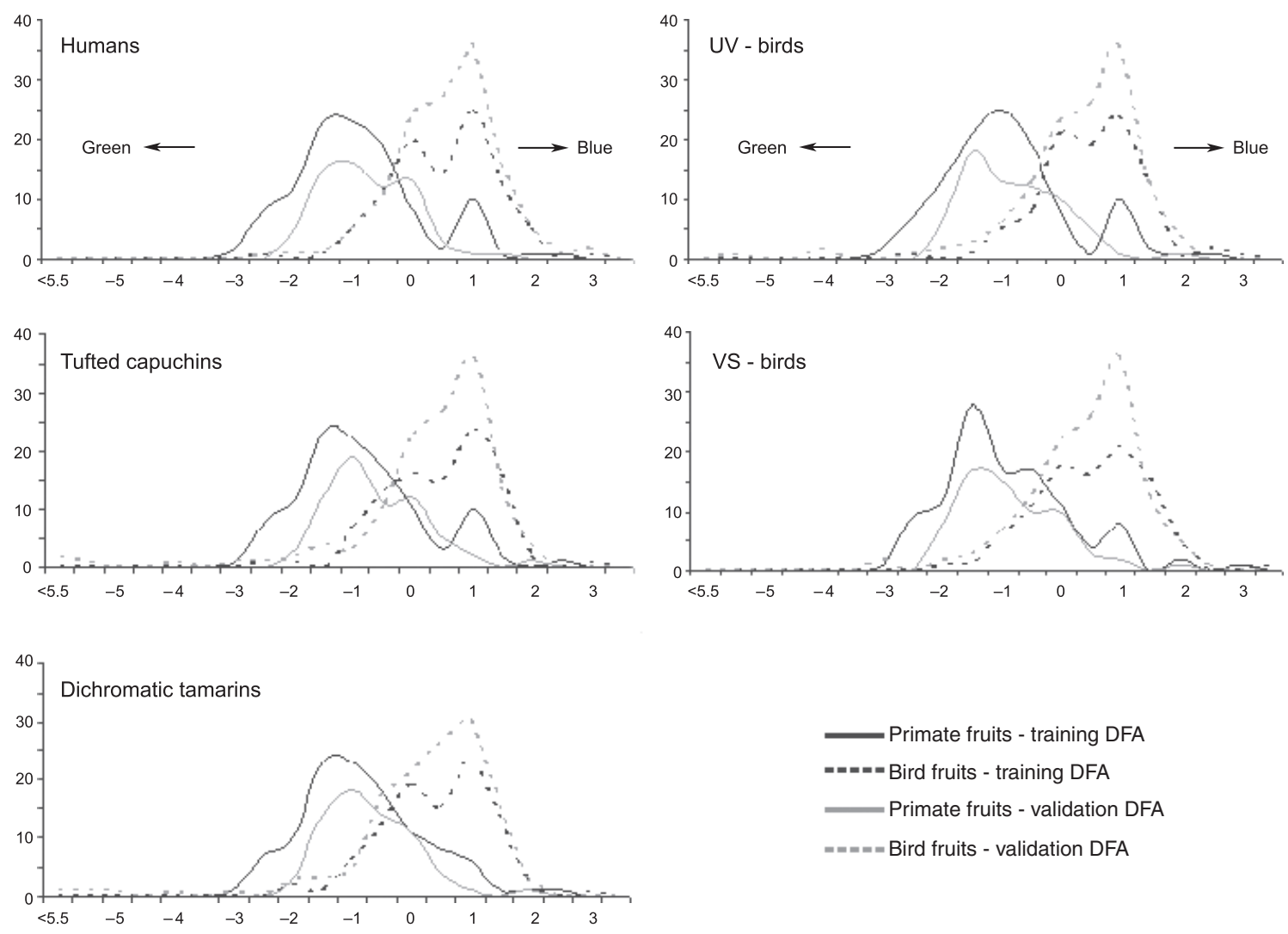

Fig. 2 Results of the Discriminant Function Analysis (DFA) based on cone excitation for the five types of frugivores tested. Black lines correspond to the "training" DFA and grey lines to the validation DFA. Dotted lines represent primate-dispersed fruits and solid lines represent bird-dispersed fruits. Primate-dispersed fruits tend to have negative scores on the linear discriminant function, which suggests that primate fruits are greener than bird fruits, which have more of a blue hue.

Table 1 (a) Mean score on LDF 1 of bird- and primate-dispersed fruits for each group of frugivore for the 'training' and the validation DFA's. (b) Loadings of the quantum catch variables for primate and bird cone sensitivities for the 'training' DFA.

\begin{tabular}{llrrr}
\hline & Humans & Tufted capuchins & Dichromatic tamarin & UV-bird \\
\hline (a) & Mean score on LDF & & & VS-bird \\
Primate fruits - 'training' & -0.675 & -0.631 & -0.604 & -0.666 \\
Bird fruits - 'training' & 0.773 & 0.723 & 0.692 & -0.690 \\
Primate fruits - validation & -0.545 & -0.423 & -0.444 & 0.790 \\
Bird fruits - validation & 0.835 & 0.461 & 0.454 & -0.579 \\
(b) & Loading on LDF & & & 0.516 \\
Short (Blue light) & 1.413 & 1.522 & 1.393 & 0.650 \\
Medium (Green light) & -2.686 & -2.407 & -2.015 & 1.437 \\
Long (Red light) & 0.672 & 0.275 & NA & -1.861 \\
\hline
\end{tabular}

DFA, Discriminant Function Analysis; LDF, Linear Discriminant Functions.

accuracy was fairly high for all vision systems tested, ranging from $79 \%$ to $82 \%$ of 110 primate-dispersed species classified correctly, and $68-75 \%$ of 96 birddispersed fruits classified correctly. The lowest classification accuracy corresponds to the dichromatic tamarin, which is expected given that these primates have only two cone types for discrimination. Primate-dispersed fruits scored on average lower on the LDF than birddispersed fruits in all analyses (Table la, Fig. 1). As the medium-wavelength variable (representing green light that primarily stimulates the medium-wavelength cone) was always highly negatively correlated with the LDF, and the short-wavelength variable (representing blue light) was always highly positively correlated with the LDF (Table 1b, Fig. 2), lower LDF scores of primatedispersed compared to bird-dispersed fruits suggest that primate fruits are perceived by all frugivore groups tested as greener than bird-dispersed fruits, which tend to reflect 
more in the blue or purple part of the spectrum. The long wavelength variable, representing red light, was much less important in discriminating between bird- and primate-dispersed fruits for all groups tested.

The validation DFA also showed significant discrimination between bird- and primate-dispersed fruits for both bird cone sensitivities (VS-bird: Wilk's $\lambda_{3,192}=0.615$, $P<0.00001$; UV-bird: Wilk's $\left.\lambda_{3,192}=0.638, P<0.00001\right)$ and all three primate cone sensitivities (humans: Wilk's $\lambda=0.6163,192, P<0.00001$; dichromatic tamarins: Wilk's $\lambda=0.687_{2,193}, P<0.00001$; Tufted capuchins: Wilk's $\lambda=0.686_{3,192}, P<0.00001$ ) (Fig. 2). Again, discrimination was significant for all different distributions of species in the 'training' and validation data sets (all $P$-values $<0.00001)$. Classification accuracy was also high, with $76-85 \%$ of 66 primate-dispersed species being classified correctly (bird and monkey peak cone sensitivities, respectively), and $73-82 \%$ of 130 bird-dispersed fruits classified correctly.

Also, the DFA performed on the subset of 77 fruits for which UV reflectance was available showed the same results reported earlier: birds (Wilk's $\lambda_{4}, 72=0.792$, $P<0.002$ ) and primates (Wilk's $\lambda_{3}, 7_{33}=0.837$, $P<0.005)$ were able to discriminate between bird- and primate-dispersed fruits. This test was carried out using only the peak cone sensitivities for the Blue Tit and for humans, as the other peak cone sensitivities yielded similar results in the analyses based on 402 plant species. For birds, the short- and medium-wavelength variables were highly correlated, albeit with different signs, with the LDF, indicating that reflectance in the blue and green parts of the spectrum was far more important than UV and red reflectance for discrimination of fruits by birds (Table 2). For primates, discrimination was mainly attributed to reflectance in the medium part of the spectrum in this subset of species.

\section{Prediction no. 2 - conspicuousness of the fruits to primates}

Contrary to the predictions under the Signal Convergence hypothesis, primate-dispersed fruits were less conspicuous (i.e. contrast less against the foliage) to

Table 2 Loadings of the quantum catch variables for primate and bird cone sensitivities for the 'training' Discriminant Function Analysis on fruit species for which UV reflectance was available.

\begin{tabular}{lcc}
\hline & \multicolumn{2}{l}{ Loading on LDF 1 } \\
\cline { 2 - 3 } Variable & Primate vision & Bird vision \\
\hline UV (Ultraviolet light) & NA & 0.396 \\
Short (Blue light) & -0.874 & -2.264 \\
Medium (Green light) & 2.486 & 2.603 \\
Long (Red light) & -0.580 & -0.521 \\
\hline
\end{tabular}

LDF, Linear Discriminant Functions. primates than were bird-dispersed fruits (Mann-Whitney $U=4,273, P<0.001, n=256)$. We are confident that the difference in contrasts is attributed to differences in fruit colour and not background colour, because we found the same pattern when fruits were contrasted against the mean reflectance of the background of all 256 species (Primates: Mann-Whitney $U=8584, P<0.001$, $n=256)$.

\section{Prediction no. 3 - phylogenetic control}

For bird cone sensitivity, after correcting the $P$-values of all the regressions run between $Y$ and each of the $X$ variables using a sequential Bonferroni correction (Rice, 1989), we obtained no significant relationship of any of the clades with the $Y$ variable at the $\alpha=0.01$ level. At the $\alpha=0.05$ level, only one clade was significant, which corresponds to Guapira opposita and Neea floribunda (Nyctaginaceae) plus Phytolacca americana (Phytolaccaceae) (hereafter, Clade I). At the $\alpha=0.1$, another two clades were significant. The first one includes Couepia guianensis and Licania latifolia (Chrysobalanaceae), Garcinia gardneriana and Tovomita umbellate (Clusiaceae), Alchornea triplinerva, Glycydendron amazonicum, Neoboutonia macrocalyx, Omphalea diandra, Sapium sebiferum and Drypetes variabilis (Euphorbiaceae), Bridelia micrantha and Margaritaria nobilis (Phyllanthaceae), Passiflora crenata (Passifloraceae), Leonia glycycarpa and Rinorea brachypetala (Violaceae), (hereafter Clade II). The second one includes all the species in the first clade named above, plus a sister clade formed by Euonymus europaea (Celastraceae) and Goupia glabra (Goupiaceae) (hereafter Clade III). For primate cone sensitivity, only Clades II and III were significant at the $\alpha=0.05$ and $\alpha=0.1$ levels. No clades were significant at the $\alpha=0.01$ level.

For bird cone sensitivity, we compared seven different models with all possible combinations of the three clades that were significant at $\alpha=0.1$ to see which combination explained best the score of plant species on the LDF (dependent variable $Y$ ). To do this, we used a maximum likelihood approach and selected the linear models based on the deviance and Aikaike's Information Criterion (AIC) (Table 3). The best models were the ones that included Clade I and either Clades II or III. Including all three clades did not improve the model significantly $(\triangle \mathrm{AIC}<2)$. Clades II and III are highly redundant, because Clade II is completely nested within Clade III (see species list earlier). Hence, we will only consider hereafter Clade II, as adding the two extra species included in Clade III does not improve the fit of the model significantly (Table 3).

For primate cone sensitivity, we compared three models including all combinations of the two clades that were significant at the $\alpha=0.05$ and $\alpha=0.1$, following the same procedure as for bird cone sensitivity (Table 3 ). Models including either Clade II, Clade III or both were not differentiable according to their AIC. This is, again, 
Table 3 Aikaike's Information Criterion (AIC) table, comparing all the possible models with Clades I, II and III to explain the dependent variable $Y$, which represents the score of all plant species on LDF 1. $\triangle \mathrm{AIC}$ represents the difference in AIC between each model and the previous one.

\begin{tabular}{lllll}
\hline & Model & Deviance & AIC & AAIC \\
\hline Bird cone & $Y$ = Clade I + Clade II & 242.99 & 583.45 & NA \\
sensitivity & $Y$ = Clade I + Clade III & 243.47 & 583.82 & 0.36 \\
& $Y$ = Clade I + Clade II & 242.67 & 585.21 & 1.39 \\
& + Clade II & & & \\
& $Y$ = Clade I & 258.82 & 593.13 & 7.92 \\
& $Y$ = Clade II & 262.84 & 595.98 & 2.85 \\
& $Y$ = Clade III & 263.24 & 596.26 & 0.28 \\
& $Y=$ Clade II + Clade III & 262.44 & 597.70 & 1.44 \\
Primate cone & $Y$ = Clade II & 264.02 & 596.81 & NA \\
sensitivity & $Y=$ Clade III & 264.14 & 596.89 & 0.08 \\
& $Y$ = Clade II + Clade III & 262.44 & 598.41 & 1.52 \\
\hline
\end{tabular}

LDF, Linear Discriminant Functions.

attributed to the great overlap between these two clades, and we will hereafter consider only Clade II.

In summary, for bird cone sensitivity under a highly conservative phylogenetic approach considering the clades that were significant at $\alpha=0.1$, we see a slight phylogenetic signal in two of the 96 clades that form the phylogeny. These two clades include 17 of the 187 species in the phylogeny. If we take a phylogenetically less conservative approach and consider only those clades that are significant at the $\alpha=0.05$ or 0.01 level (which would be statistically more conservative), we find that only one clade with three species or none show any influence in the discrimination between bird- and primate-dispersed species, respectively. For primate cone sensitivity, we find that only one clade of 17 species influences the discrimination between bird- and primatedispersed species when considering clades significant at $\alpha=0.05$ and $\alpha=0.1$ levels.

The results from the EPCA are qualitatively similar, as we show that phylogenetic signal does not seem to be very important. Bird- and primate-dispersed fruits converged to similar colours, both when analysed from the primate and from the bird perspectives (Supporting Information).

\section{Discussion}

Our results generally support the first prediction of the Signal Convergence hypothesis, which states that frugivores should be able to discriminate between the colour of fruits dispersed by them and those of fruits dispersed by other frugivores. Based on our vision models of three different primates and two types of birds, we conclude that these frugivores should be able to differentiate between fruits typically dispersed by primates and those dispersed by birds. This is important because fruit colour is thus an informative trait that can be used by frugivores to find and choose fruits.
Fruits of many plants are consumed and dispersed by both birds and primates. Disperser overlap does not contradict the Signal Convergence, nor the Dispersal Syndrome hypotheses, because neither implies exclusive consumption by one frugivore type. If syndromes are an evolutionary response to seed dispersers, they should result from plants responding to the primary selective pressure, in this case, the seed dispersers that most improve their fitness, even though other less efficient frugivores may still feed on the same plant. Even if the relative fitness benefit conveyed from one seed disperser type is small compared to that of other dispersers, it may lead to the evolution of distinct phenotypic traits associated with the best disperser (see Chittka et al., 2001 for a similar argument on pollination). Thus, our result of signal divergence in bird- and primate-dispersed species holds even though - and not because - we classified plants according to their primary disperser as either primate- or bird-dispersed species. It would be interesting to see where species dispersed by both birds and primates score on the discriminant function. We would predict that they would mostly fall in the area of overlap of birdand primate-dispersed fruits, as birds and primates perceive them (Fig. 2). We could not test this in this manuscript, because the data we used were from studies that either focused on bird dispersal or on primate dispersal. Hence, for the great majority of species, only one type of disperser was recorded, and the very few data on mixed dispersal are anecdotal and may yield biased results.

Moreover, we tested only colour as a signal associated with disperser type. Thus, although we believe colour is one of the most important cues used by diurnal frugivores to find fruits, we acknowledge that it is certainly not the only one. The fact that the colour of, for example, some bird-dispersed fruits falls well within the range of a primate's visual perception does not mean that bird fruits are necessarily easy to find by primates, as primates may need a combination of cues to find fruits, such as colour, odour and texture (Dominy et al., 2001; Dominy, 2004). If we could include other cues used by these frugivores to find fruits in our model, we would likely obtain sharper discrimination.

Our second prediction, that the fruits dispersed by a frugivore should be more conspicuous to that frugivore than fruits not dispersed by them, was not supported by our data. Primate fruits are less conspicuous to primates than bird fruits. We propose several alternative explanations for this result dividing them into 'adaptive' and 'nonadaptive' explanations from the perspective of plant-frugivore communication. The two adaptive explanations are consistent with the Signal Convergence hypothesis. First, it is possible that, whereas birds have such well developed colour vision and may be primarily visual foragers, primates rely to a larger extent on other cues besides colour, such as odour, size and texture of fruits to assess their edibility (Dominy, 2004; Laska et al., 
2007). As such, there might be a stronger selective pressure on fruit conspicuousness by birds than by primates. If this is the case, we predict that bird-dispersed fruits should be more conspicuous to birds than are primate-dispersed fruits. And second, it is also possible that limiting conspicuousness to chromatic contrasts is simplistic. Sumner \& Mollon (2000b) argue that, at equal contrast between background and fruit colour, larger fruits are more conspicuous to primates simply because of their size. Consequently, large-fruited plants that are typically dispersed by mammals may not be under as strong selective pressure as small-fruited plants to maximize their conspicuousness. Under this hypothesis, we predict an inverse relationship between the LDF score and fruit size among primate-dispersed species, because LDF is negatively associated with reflectance of green light, which is less conspicuous against the foliage. This is similar to what Sumner \& Mollon (2000b) showed when they correlated fruit size with reflectance at longer wavelengths. However, their negative correlation can also be explained by the effect of smaller, more visual frugivores, such as birds feeding on smaller fruits, as predicted by the Dispersal Syndrome hypothesis (Lomáscolo et al., 2008), rather than the sole effect of selection on conspicuousness by one type of frugivore (Sumner \& Mollon, 2000b). Whatever the ultimate explanation for this correlation, we suggest that conspicuousness to frugivores should be measured as a function of fruit size, in addition to the contrast against the background.

The next explanations we discuss consider the roles of colour that are independent of plant-frugivore communication such as defence against pathogens or seed predators, as well as physiological or phylogenetic constraints (Willson \& Whelan, 1990). First, anthocyanins, the pigments responsible for conspicuous red and black fruit colours, reduce fungal growth (Schaefer et al., 2008 b). The prevalence of red and black fruits (Wheelwright \& Janson, 1985) may thus be, at least partly, explained by the antimicrobial properties of these pigments and may not be independent from the nutritional value of fruit resources. For example, blue fruits that, according to our results, indicate that bird dispersal is associated with high sugar contents (Schaefer \& Schmidt, 2004). If pigments are primarily selected for their defensive function, colour signals should be considered an exaptation (Gould \& Vrba, 1982) instead of an adaptation to endozoochory. And second, colour evolution may be limited by phylogenetic constraints. Fruit traits may be highly conserved (Jordano, 1995), probably attributed to the long generation time of plants in comparison with seed dispersers (Herrera, 1985). However, our phylogenetic comparative analyses suggest that similar colours among species dispersed by primates and those dispersed by birds are mostly the result of convergence rather than shared common ancestry. Even when we used a highly conservative phylogenetic approach, i.e. when we considered clades that seemed to be marginally significant in determining the scores on the LDF, we saw that at most two of 96 clades in the phylogeny showed any phylogenetic signal at all, both for bird and primate cone sensitivity. In other words, $97.9 \%$ of the clades show no phylogenetic signal in the colour perceived by birds or primates. This is consistent with the evidence that fruit colouration seems to be evolutionarily less constrained than fruit morphology and nutritional contents (A. Valido et al. unpublished manuscript). This is also supported by the apparent convergence of fruit colours in the 81 primate-dispersed and the 106 bird-dispersed fruits analysed with EPCA (Supporting Information).

Although primates have sometimes been merged with birds in a bird-primate syndrome because no differences in colour were found between fruits dispersed by these two groups of frugivores (Gautier-Hion et al., 1985), we show that, although primates and birds overlap in the colour of the fruits they consume, primates disperse more green fruits than do birds, and birds disperse more blue fruits than do primates. These results match those of earlier studies in fruit choice by frugivores (Janson, 1983; Wheelwright \& Janson, 1985). Yet, our discriminant analysis is a conservative analysis, because restricting colour vision to three cones in birds - owing to the exclusion of UV - underestimates avian discrimination abilities. However, even though reflectance in the UV part of the spectrum has been shown to increase detectability for birds (Altshuler, 2001; Schaefer et al., 2006, 2007), our test of 77 plant species shows that UV reflectance in fruit may not be reliably associated with bird dispersal. This conjecture is consistent with experimental evidence showing no discrimination among fruits with or without UV-reflecting bloom (Willson \& Whelan, 1989).

Although we cannot be sure that our model exactly represents how birds and primates perceive colour, as colour vision entails much more than cone excitation, our model still provides an objective approximation of the basic input of colour vision by these frugivores. This is the first study that addresses the Signal Convergence hypothesis in a fruit-frugivore system, using such an objective measure of colour and such a large data set of reflectance spectra (the largest analysed to date).

Colour is an important mediator in plant-animal communication (this study, Johnson \& Steiner, 2000; Schaefer et al., 2004; Chittka \& Raine, 2006), and we believe that learning the signalling role of colour will help understand how animals and plants affect each other's fitness and evolution. This can only be performed by using different vision models that incorporate the receptive and cognitive processes of the receivers, which adds much needed objectivity in the assessment of how plants signal to animals (Schaefer et al., 2004). 


\section{Acknowledgments}

We thank Doug Levey for all his guidance and comments on this manuscript. We also thank Ignacio Paz Posse and Veronika Schaefer for their support while writing this manuscript, and beyond. Ignacio Paz Posse also helped with figure preparation. Valuable comments from Rebecca Kimball, Ben Bolker, Mike Miyamoto, Emilio Bruna, Jacqui Shykoff and one anonymous reviewer improved this manuscript. Norberto Giannini, Eric Dyreson, Rebecca Kimball and Romina Vidal-Russell greatly helped with the phylogenetic comparative analysis. SBL was supported by NSF grant DEB-0513649, the Department of Zoology, UF, and a fellowship from the College of Liberal Arts and Sciences, UF; HMS was supported by a DFG grant Scha 1008/4-1.

\section{References}

Altshuler, D.L. 2001. Ultraviolet reflectance in fruits, ambient light composition and fruit removal in a tropical forest. Evol. Ecol. Res. 3: 767-778.

Ben-Tal, Y. \& King, R.W. 1997. Environmental factors involved in colouration of flowers of Kangaroo Paw. Sci. Hortic. 72: $35-$ 48 .

Blüthgen, N., Menzel, F., Hovestadt, T. \& Fiala, B. 2007. Specialization, constraints, and conflicting interests in mutualistic networks. Curr. Biol. 17: 341-346.

Bowmaker, J.K. \& Hunt, D.M. 2006. Evolution of vertebrate visual pigments. Curr. Biol. 16: R484-R489.

Burns, K.C. 2005. Does mimicry occur between fleshy-fruits? Evol. Ecol. Res. 7: 1067-1076.

Burns, K.C. \& Dalen, J.L. 2002. Foliage color contrasts and adaptive fruit color variation in a bird-dispersed plant community. Oikos 96: 463-469.

Campbell, O.W. \& Donoghue, M.J. 2004. Phylomatic: tree assembly for applied phylogenetics. Mol. Ecol. 5: 181-183.

Chittka, L. \& Raine, N.E. 2006. Recognition of flowers by pollinators. Curr. Opin. Plant Biol. 9: 428-435.

Chittka, L., Spaethe, J., Schmidt, A. \& Hickelsberger, A. 2001. Adaptation, constraint, and chance in the evolution of flower color and pollinator color vision. In: Cognitive Ecology of Pollination (L. Chittka \& J.D. Thomson, eds), pp. 106-126. University Press, Cambridge.

Clark, C.J., Poulsen, J.R. \& Parker, V.T. 2001. The role of arboreal seed dispersal groups on the seed rain of a lowland tropical forest. Biotropica 33: 606-620.

Dominy, N.J. 2004. Fruits, fingers, and fermentation: the sensory cues available to foraging primates. Integr. Comp. Biol. 44: 295-303.

Dominy, N.J., Lucas, P.W., Osorio, D. \& Yamashita, N. 2001. The sensory ecology of primate food perception. Evol. Anthropol. 10: $171-186$.

Dyreson, E. \& Maddison, W.P. 2006. Rhetenor Package for Morphometrics. Mesquite 2.01.

Endler, J.A. 1992. Signals, signal conditions, and the direction of evolution. Am. Nat. 139: S125-S153.

Endler, J.A. \& Mielke, P.W. Jr 2005. Comparing entire color patterns as birds see them. Biol. J. Linn. Soc. Lond. 86: 405431.
Feinsinger, P., Tiebout, H.M. \& Young, B.E. 1991. Do tropical bird-pollinated plants exhibit density-dependent interactions field experiments. Ecology 72: 1953-1963.

Fischer, K.E. \& Chapman, C.A. 1993. Frugivores and fruit syndromes - differences in patterns at the genus and species level. Oikos 66: 472-482.

Forsyth, S.A. 2003. Density-dependent seed set in the Haleakala silversword: evidence for an Allee effect. Oecologia 136: 551-557. Gautier-Hion, A., Duplantier, J.M., Quris, R., Feer, F., Sourd, C., Decoux, J.P., Dubost, G., Emmons, L., Erard, C., Hecketsweiler, P., Moungazi, A., Roussilhon, C. \& Thiollay, J.M. 1985. Fruit characters as a basis of fruit choice and seed dispersal in a tropical forest vertebrate community. Oecologia 65: 324-337.

Giannini, N.P. 2003. Canonical phylogenetic ordination. Syst. Biol. 52: 684-695.

Goloboff, P.A., Carpenter, J.M., Arias, J.S. \& Esquivel, D.R.M. 2008. Weighting against homoplasy improves phylogenetic analysis of morphological datasets. Cladistics 24: 758-773.

Gould, S.J. \& Vrba, E.S. 1982. Exaptation - a missing term in the science of form. Paleobiology 8: 4-15.

Gumbert, A., Kunze, J. \& Chittka, L. 1999. Floral colour diversity in plant communities, bee colour space and a null model. Proc. R. Soc. Lond. B Biol. Sci. 266: 1711-1716.

Håstad, O. \& Ödeen, A. 2008. Different ranking of Avian Colors predicted by modeling of retinal function in humans and Birds. Am. Nat. 171: 831-838.

Herder, F., Nolte, A.W., Jobst, P., Schwarzer, J., Hadiaty, R.K. \& Schliewen, U.K. 2006. Adaptive radiation and hybridization in Wallace's Dreamponds: evidence from sailfin silversides in the Malili Lakes of Sulawesi. Proc. R. Soc. Lond. B Biol. Sci. 273: 2209-2217.

Herrera, C.M. 1985. Determinants of plant-animal coevolution the case of mutualistic dispersal of seeds by vertebrates. Oikos 44: 132-141.

Herrera, C.M. 1987. Vertebrate-dispersed plants of the Iberian Peninsula - a study of fruit characteristics. Ecol. Monogr. 57: 305-331.

Janson, C.H. 1983. Adaptation of fruit morphology to dispersal agents in a Neotropical forest. Science 219: 187-189.

Johnson, S.D. \& Steiner, K.E. 2000. Generalization versus specialization in plant pollination systems. Trends Ecol. Evol. 15: 140-143.

Jordano, P. 1995. Angiosperm fleshy fruits and seed dispersers a comparative analysis of adaptation and constraints in plantanimal interactions. Am. Nat. 145: 163-191.

Kalko, E.K.V., Herre, E.A. \& Handley, C.O. 1996. Relation of fig fruit characteristics to fruit-eating bats in the New and old World tropics. J. Biogeogr. 23: 565-576.

Kelber, A., Vorobyev, M. \& Osorio, D. 2003. Animal colour vision - behavioural tests and physiological concepts. Biol. Rev. 78: 81-118.

Knight, R.S. \& Siegfried, W.R. 1983. Inter-relationships between type, size and colour of fruits and dispersal in Southern African trees. Oecologia 56: 405-412.

Korine, C., Kalko, E.K.V. \& Herre, E.A. 2000. Fruit characteristics and factors affecting fruit removal in a Panamanian community of strangler figs. Oecologia 123: 560-568.

Laska, M., Freist, P. \& Krause, S. 2007. Which senses play a role in nonhuman primate food selection? A comparison between squirrel monkeys and spider monkeys. Am. J. Primatol. 69: 282-294. 
Lomáscolo, S., Speranza, P. \& Kimball, R. 2008. Correlated evolution of fig size and color supports the Dispersal Syndromes hypothesis. Oecologia 156: 783-796.

Lord, J.M., Markey, A.S. \& Marshall, J. 2002. Have frugivores influenced the evolution of fruit traits in New Zealand? In: Seed Dispersal and Frugivory: Ecology, Evolution and Conservation (D.J. Levey, W.R. Silva \& M. Galetti, eds), pp. 55-68. CABI Publishing, Wallingford.

Maddison, D.R. \& Maddison, W.P. 2007. Mesquite: A Modular System for Evolutionary Analysis. Version 2.01.

Ödeen, A. \& Hastad, O. 2003. Complex distribution of avian color vision systems revealed by sequencing the SWS1 opsin from total DNA. Mol. Biol. Evol. 20: 855-861.

Osorio, D. \& Vorobyev, M. 1996. Colour vision as an adaptation to frugivory in primates. Proc. R. Soc. Lond. B Biol. Sci. 263: 593-599.

van der Pijl, L. 1961. Ecological aspects of flower evolution .2. Zoophilous flower classes. Evolution 15: 44.

van der Pijl, L. 1969. Principles of Dispersal of Higher Plants. Academic Press, Orlando, Fla.

de Pinho Werneck, F., Colli, G.R. \& Vitt, L.J. 2009. Determinants of assemblage structure in Neotropical dry forest lizards. Austral Ecol. 34: 97-115.

Poulsen, J.R., Clark, C.J., Connor, E.F. \& Smith, T.B. 2002. Differential resource use by primates and hornbills: implications for seed dispersal. Ecology 83: 228-240.

R Development Core Team 2008. A language and environment for statistical computing, $\mathrm{R}$ Foundation for Statistical Computing, Vienna, Austria. ISBN 3-900051-07-0, URL http://www.R-project.org.

Regan, B.C., Julliot, C., Simmen, B., Vienot, F., CharlesDominique, P. \& Mollon, J.D. 2001. Fruits, foliage and the evolution of primate colour vision. Philos. Trans. R. Soc. Lond. B Biol. Sci. 356: 229-283.

Rice, W.R. 1989. Analyzing Tables of Statistical Tests. Evolution 43: 223-225.

Ridley, H.N. 1930. The Dispersal of Plants Throughout the World. L. Reeve 8 Co. Ltd., Ashford.

Schaefer, H.M. \& Schmidt, V. 2004. Detectability and content as opposing signal characteristics in fruits. Proc. R. Soc. Lond. B Biol. Sci. 271: S370-S373.

Schaefer, H.M., Schaefer, V. \& Levey, D.J. 2004. How plantanimal interactions signal new insights in communication. Trends Ecol. Evol. 19: 577-584.

Schaefer, H.M., Levey, D.J., Schaefer, V. \& Avery, M.L. 2006. The role of chromatic and achromatic signals for fruit detection by birds. Behav. Ecol. 17: 784-789.

Schaefer, H.M., Schaefer, V. \& Vorobyev, M. 2007. Are fruit colors adapted to consumer vision and birds equally efficient in detecting colorful signals? Am. Nat. 169: S159-S169.

Schaefer, H.M., McGraw, K.J. \& Catoni, C. 2008a. Birds use fruit colour as honest signal of dietary antioxidant rewards. Funct. Ecol. 22: 303-310.

Schaefer, H.M., Rentzsch, M. \& Breuer, M. 2008b. Anthocyanins reduce fungal growth in fruits. Nat. Prod. Commun. 3: 12671272.

Schlick-Steiner, B.C., Steiner, F.M., Moder, K., Seifert, B., Sanetra, M., Dyreson, E., Stauffer, C. \& Christian, E. 2006. A multidisciplinary approach reveals cryptic diversity in Western Palearctic Tetramorium ants (Hymenoptera: Formicidae). Mol. Phylogenet. Evol. 40: 259-273.

Schmidt, V., Schaefer, H.M. \& Winkler, H. 2004. Conspicuousness, not colour as foraging cue in plant-animal signaling. Oikos 106: 551-557.

Sharma, S., Jhala, Y. \& B., S.V. 2005. Identification of individual tigers (Panthera tigris) from their pugmarks. J. Zool. 267: 9-18.

Sumner, P. \& Mollon, J.D. 2000a. Catarrhine photopigments are optimized for detecting targets against a foliage background. J. Exp. Biol. 203: 1963-1986.

Sumner, P. \& Mollon, J.D. 2000b. Chromaticity as a signal of ripeness in fruits taken by primates. J. Exp. Biol. 203: 19872000.

Voigt, F.A., Bleher, B., Fietz, J., Ganzhorn, J.U., Schwab, D. \& Bohning-Gaese, K. 2004. A comparison of morphological and chemical fruit traits between two sites with different frugivore assemblages. Oecologia 141: 94-104.

Vorobyev, M. \& Osorio, D. 1998. Receptor noise as a determinant of colour thresholds. Proc. R. Soc. Lond. B Biol. Sci. 265: 351-358.

Waser, N.M., Chittka, L., Price, M.V., Williams, N.M. \& Ollerton, J. 1996. Generalization in pollination systems, and why it matters. Ecology 77: 1043-1060.

Wheelwright, N.T. \& Janson, C.H. 1985. Colors of fruit displays of bird-dispersed plants in two tropical forests. Am. Nat. 126: 777-799.

Willson, M.F. \& Whelan, C.J. 1989. Ultraviolet reflectance of fruits of vertebrate-dispersed plants. Oikos 55: 341-348.

Willson, M.F. \& Whelan, C.J. 1990. The evolution of fruit color in fleshy-fruited plants. Am. Nat. 136: 790-809.

Willson, M.F., Irvine, A.K. \& Walsh, N.G. 1989. Vertebrate Dispersal Syndromes in Some Australian and New-Zealand Plant-Communities, with Geographic Comparisons. Biotropica 21: 133-147.

\section{Supporting information}

Additional Supporting Information may be found in the online version of this article:

Figure S1. Phylogenetic tree of 187 species done with Phylomatic. This tree 52 structure was used as the basis of our phylogenetic comparative analyses.

Table S1. Loadings of the quantum catch variables for primate and bird cone sensitivities for the EPCA.

As a service to our authors and readers, this journal provides supporting information supplied by the authors. Such materials are peer-reviewed and may be reorganized for online delivery, but are not copy-edited or typeset. Technical support issues arising from supporting information (other than missing files) should be addressed to the authors.

Received 30 March 2009; revised 7 December 2009; accepted 10 December 2009 